Stefan Kühl

\title{
Organisationssoziologie. Ein Ordnungs- und Verortungsversuch
}

In vielen Bindestrich-Soziologien lässt sich die Tendenz beobachten, sich nicht nur als Experte für ein Spezialgebiet der Soziologie zu begreifen, sondern vom eigenen Kategoriengerüst aus eine Gesellschaftsinterpretation anzubieten. ${ }^{.}$Die Techniksoziologie versteht sich häufig nicht nur als Disziplin zur Rekonstruktion von Technikentwicklungen, sondern hat den Anspruch gesamtgesellschaftliche Entwicklungen zu erklären (siehe z. B. Latour 1995). In der politischen Soziologie lässt sich ein Trend beobachten, sich nicht nur auf die Analyse des Funktionssystems der Politik zu beschränken, sondern eine vom Begriff des Politischen ausgehende eigene Gesellschaftsanalyse vorzuschlagen. Die Arbeitssoziologie bietet nicht nur ein Verständnis von Arbeit an, sondern es wird eine eigene Gesellschaftstheorie aufgestellt, in der auch politische, kulturelle und wissenschaftliche Entwicklungen auf die Schlüsselkategorie Arbeit zurückgeführ werden. Aus der Organisationssoziologie kommend gibt es einen Trend, sich nicht mit der Beschreibung der Funktionsweise von Organisationen zu begnügen, sondern die Entwicklung zu einer Organisationsgesellschaft festzustellen.

Je stärker sich die Soziologie in Bindestrichsoziologien ausdifferenziert, desto stärker scheint die Verlockung in diesen zu sein die eigene Perspektive, den eigenen Kategorienkatalog und die eigenen Theorien zur Analyse der Gesamtgesellschaft auszuweiten. Hier reproduziert die Soziologie in ihren eigenen Differenzierungen eine Entwicklung, die sich auf allgemeinerer Ebene bei den Reflektionswissenschaften der einzelnen Funktionssystemen beobachten lässt (vgl Beck 1982: 16; Kieserling 2000: 58f.). Beispielsweise gibt es, wie André Kieserling herausgestellt hat, die Tendenz der Rechtswissenschaft sich nicht nur als Instrument zur Analyse des Rechtssystems zu begreifen, sondern die Gesamtgesellschaft von der Perspektive des Rechts aus zu betrachten. Wenn die Wissenschaft feststellt, so ein anderes Beispiel, dass es Systeme gibt, die keine Verwissenschaftlichung ihrer Handlungen vertragen, dann wird dies von der Wissenschaft als Pathologie, Irrationalität oder Abweichung begriffen und nicht als legitimer Ausdruck von gesellschaftlicher Differenzierung.

Der expansive Anspruch, der vielen Bindestrichsoziologien innewohnt, lässt sich besonders bei der Arbeits- und bei der Organisationssoziologie beobachten

I Besten Dank an Ulrich Beck, Norman Braun, Andre Kjeserling und Veronika Tacke für hilfreiche Kommentare zu einer früheren Fassung des Manuskripts. 
Die beiden Soziologien, die sich am intensivsten mit Unternehmen, Gewerkschafien und Verwaltungen beschäftigen, die Arbeitssoziologie und die Organisationssoziologie, tendieren dazu, ihre eigenen Zentralkategorien "Arbeit" bzw. „Organisation“ zu überschätzen und sich so blinde Flecken nicht nur in Bezug auf ihre Gesellschaftstheorie, sondern auch in Bezug auf ihren eigentlichen Gegenstand einzuhandeln. Es ist deswegen ein an Bindestrichsoziologien im allgemeinen und an die Arbeits- bzw. die Organisationssoziologie im besonderen anzulegendes Qualitätskriterium, inwiefern die Grenzen des eigenen Kategorienkatalogs bei der Formulierung einer Gesellschaftstheorie mitreflektiert werden und wie vor dem Hintergrund dieser Reflektion der eigenen Grenzen das Verhältnis des eigenen Gegenstands (Arbeit, Technik, Organisation etc.) zur Gesellschaft bestimmt wird.

\section{Die Zentralkategorie der Arbeit und der expansive Anspruch der \\ Arbeitssoziologie}

Die Arbeits-, Industrie- und Betriebssoziologie hatte den Anspruch, mehr zu sein als eine der Bindestrichsoziologien (vgl. z. B. Bahrdt 1982; Beckenbach 1991; ausführlich Daheim/Schönbauer 1993). Sie verstand sich als eine Soziologie, über deren Analyse der Produktionsverhältnisse nicht nur die Zustände in den Betrieben, sondern auch gesamtgesellschaftliche Verhältnisse erklärt werden können. Mit der Postulierung einer Arbeitsgesellschaft oder einer - häufig synonym verwendeten - Industriegeselischaft wurde der Anspruch erhoben, über dje Zentralkategorie Arbeit nicht nur Verhältnisse in Betrieben, sondern in der Gesellschaft insgesamt beschreiben zu können (vgl. Ostner 2001: 57ff.) Über die Kategorie der Arbeit hatte die Arbeitssoziologie - und das war sicherlich eine ihrer Stärken - eine überzeugende Anschlussstelle an die Gesellschaftstheorie und lief in dieser Konzeptualisierung nicht Gefahr, die Beziehung zwischen den Verhältnissen in den Betrieben und gesamtgesellschaftlichen Fragen zu negieren (vgl. Tacke/Wehrsig 1992: 220).

Aufgrund dieser Fokussierung gab es eine deutliche theoretische Präferenz der Arbeits-, Betriebs- und Industriesoziologie in der Nachkriegszeit. Gerhard Brandt stellt zu Recht heraus, dass die Arbeits-, Betriebs- und Industriesoziologie nach dem Zweiten Weltkrieg aufs engste der Marx'schen Gesellschafts- und Geschichtstheorie verhaftet war. Kategorial gesehen hielten sich die arbeits-, betriebs- und industriesoziologischen Forschungsansätze fast durchweg im objektivistischen Bezugsrahmen der Kritik der politischen Ökonomie (vgl. Brandt 1990: 254, 263). Die Debatte über neue Unternehmensformen, sicherlich ein Haupthema bei der Analyse der Arbeitsorganisation in Unternehmen, wurde als Teil einer Auseinandersetzung zwischen durch „objektive "Interessen geprägten antagonistischen Lagern begriffen. Auf der einen Seite der Auseinandersetzung über neue Unternehmensformen finden sich diejenigen, die aufgrund ihres $\mathrm{Ka}$ pitalbesitzes über fremde Arbeitskraft verfügen können. Auf der anderen Seite sind diejenigen, die nichts zu verkaufen haben als ihre Arbeitskraft (siehe Bardmann 1994: $222 \mathrm{ff}$. für eine ausführliche ideengeschichtliche Darstellung)

Die Gefahr für eine so verstandene Arbeits-, Betriebs- und Industriesoziologie ist erstens eine Überstrapazierung ihres eigenen Kategoriengerüsts bei einer Interpretation der Gesamtgesellschaft und zweitens ein nur unscharfer Fokus auf die Prozesse in den einzelnen Unternehmen.

Zu eins: Ein Effekt war, dass die Arbeits-, Betriebs- und Industriesoziologie Beziehungen zwischen Arbeitgeber und Arbeitnehmer nicht als vorrangig organisationsinterne Beziehung behandelte, sondern daraus eine zentrale gesamtgesellschaftliche Konfliktlinie rekonstruierte. Die Stellung der Arbeitenden im industriellen Arbeitsprozess bestimmt, so die Annahme, nicht nur seine Rolle im Betrieb, sondern auch in der Gesellschaft als Ganzes (vgl. dazu beispielsweise Kreckel 1983; Ostner 2001). Auch wenn diese Beschreibung für historische Situationen in einigen Industriestaaten im späten neunzehnten und frühen zwanzigsten Jahrhundert richtig gewesen sein mag, so wird doch mit einer zunehmenden Ausdifferenzierung der Gesellschaft in eigenständige Funktionssysteme diese Leitdifferenz fragwürdig. Bei aller Wichtigkeit von Tarifauseinandersetzungen zwischen Arbeitgebern und Arbeitnehmern für interne Beziehungen in Organisationen scheint sich diese Auseinandersetzung nicht als Ordnungsschema für die Gesamtgesellschaft zu eignen (vgl. Offe 1984).

Zu zwei: Der Preis für die über den Marxismus hergestellte gesellschaftstheoretische Anbindung der Arbeits-, Industrie- und Betriebssoziologie war, dass es keinen systematischen „Ort" für die Analyse des Eigenlebens von Organisationen gab. Selbst Forschungsrichtungen wie dem betriebsstrategischen Ansatz gelang es nicht, eine umfassende eigenständige Organisationsperspektive zu entwickeln. Letztlich wurden „unternehmerische Autonomiestrategien" so verstanden, dass die zur „Verwirklichung des Produktionsziels notwendigen ökonomischen, technischen und sozialen Strukturen des Betriebes" so organisiert werden, dass der Betrieb nicht auf Leistungen angewiesen ist, deren Verwertung mit „,betriebsfremden Auflagen" für das betriebliche Herrschaftssystem verbunden ist (vgl. Altmann/Bechtle 1971: 30). Durch diese Konzeptualisierung wurde dem Unternehmensmanagement zwar innerhalb der ökonomischen Verhältnisse Handlungsspielraum zur Herrschaftssicherung eingeräumt, letztlich aber die Determinierung der betrieblichen Abläufe durch die ökonomischen Verhältnisse beibehalten (siehe auch Altmann et al. 1982).

Von wenigen theoretisch interessanten Arbeiten (z.B. Böhle 1994; Voß/Pongratz 1998; Wolf 1999) abgesehen, hat die Hauptströmung der deutschsprachigen Arbeits-, Industrie- und Betriebssoziologie einen explizit marxistischen Zugang zur Analyse von Betrieben und Gesellschaft fallen gelassen. Mit diesem wohl auch auf politische Moden und auf die Bedingungen öffentlich geförderter Forschung zurückzuführenden Abschied von einer marxistischen Theorieentwicklung scheint die Arbeits-, Industrie- und Betriebssoziologie zurzeit sowohl die Verortung in der allgemeinen Soziologie als auch die präzise theoretische Bestimmung ihres Gegenstandes zu verlieren. Die Arbeits-, Betriebs- und 
Industriesoziologie droht in ein theoretisches und konzeptuelles Vakuum zu fallen, aus dem sie sich vielleicht nur durch eine Rückbesinnung auf den Marxismus oder durch eine grundlegende Neubestimmung des Verhältnisses von Arbeit zur Gesellschaft einerseits und zur Organisation andererseits befreien kann. Letzteres würde jedoch aller Wahrscheinlichkeit nach in eine Organisationssoziologie führen, die mit der Arbeits-, Industrie- und Betriebssoziologie der siebziger, achtziger aber auch teilweise der neunziger Jahre wenig zu tun hat.

\section{Die Organisationsgesellschaft und wie steht es um Organisation als}

\section{Zentralkategorie der Soziologie}

Die Organisationssoziologie tritt explizit, häufiger jedoch nur implizit mit dem Anspruch an, die Fokussierung der Arbeits-, Industrie- und Betriebssoziologie auf eine Zentralkategorie der Arbeit zu überwinden und damit das Phänomen der Organisation stärker in den Mittelpunkt einer Analyse zu rücken. Für die Organisationssoziologie besteht dabei die Herausforderung darin, den eigenen Organisationsbegriff an eine Gesellschaftstheorie anzuschließen, ohne diesen jedoch zur Zentralkategorie zur Analyse der Gesamtgesellschaft auszudehnen. Vermutlich seit Claude Henry Saint-Simon, spätestens jedoch seit Max Weber gibt es eine Tradition in der Soziologie, Gesellschaften von ihren Organisationen her zu begreifen. In „Du système industriel“, einer der ersten soziologischen Arbeiten überhaupt, vergleicht Saint-Simon (1964) die französische Gesellschaft mit einer "großen Manufaktur" und bezeichnet mit seinem Begriff des ,entreprise industriel" letztlich auch die Gesellschaft selbst. Ähnlich verschwimmt auch bei Max Webers (1972) Analyse der Entstehung eines ,stahlharten Gehäuse der Hörigkeit" Organisations- und Gesellschaftsanalyse (vgl. theoriegeschichtlich besonders interessant Schluchter 1985).

Der Effekt der Anlehnung einer Gesellschaftstheorie an die Funktionsweise von Organisationen führte besonders unter dem Eindruck des Machtzuwachses von Großunternehmen und der Ausdehnung des Staatssozialismus nach dem Zweiten Weltkrieg zu soziologischen Konzepten, die das Handeln von Individuen in der Gesellschaft ähnlich behandeln wie das Handeln in organisatorischen Kontexten. James Burnham (1948) beispielsweise stellt die Ablösung des Kapitalismus durch eine „Management-Gesellschaft ${ }^{\text {" fest }}$ und leitet seine Gesellschaftstheorie aus der Funktionsweise von Unternehmen nach dem Zweiten Weltkrieg ab. William H. Whyte (1958) geht davon aus, dass die Machtzusammenballung in Großunternehmen zu einer wachsenden Macht der Bürokratien führt und dass es - ähnlich wie schon von Burnham herausgestellt - zu der Ausbildung einer "Managementhierarchie" in der Gesellschaft kommt. Der Mensch wird nicht nur in seiner Tätigkeit im Unternehmen, sondern auch in seinem Leben als Freund, Ehepartner oder Kirchenmitglied zum „Organization Man ". Die Argumentation Whytes weist Ähnlichkeiten mit den Thesen Theodor W. Adornos über das "Ende des Individuums" in einer sich zunehmend bürokratisierenden Welt auf. Im Kontakt mit formalen Organisationen haben sich, so Adorno, sowohl die Mitglieder in Organisationen als auch die Kunden und Klienten der „Apparatur" immer ähnlicher gemacht (vgl. Adorno 1954). ${ }^{2}$

Wenn man die Arbeiten in der Tradition von Saint-Simon und Weber als Zeitdiagnosen behandelt, können sie interessante Fragen nach der Rolle der Organisation in der modernen Gesellschaft eröffnen. Sie können darauf aufmerksam machen, dass Organisationen ein Typus sozialer Systeme sind, der erst in der Moderne eine große Prominenz erreicht hat. Die Arbeiten von Bumham, Whyte oder Adorno lassen sich als zeitdiagnostisch zu nutzende Indikatoren für die Rolle von Organisationen in der Gesellschaft begreifen. Zwar lassen sich mit Shmuel Eisenstadt (1963) bereits im China der Han-Dynastie, im MogulImperium in Indien oder im Persien der Sassaniden bürokratisierte Patrimonialstaaten feststellen, aber erst in der Moderne bilden sich Organisationen als eigenständiger Systemtypus aus, der viele soziale Räume erfasst (vgl. auch Coleman 1993:2)

Als Sozialtheorien, die den Anspruch erheben, als theoretisches Instrumentarium soziologischer Analysen zu dienen, drohen die Ansätze jedoch zu zwei theoretischen Kurzschlüssen zu führen, die in beiden Formen letztlich in der vorschnellen Konstatierung einer Organisationsgesellschaft enden (vgl. Tacke 2001).

Der erste theoretische Kurzschluss besteht darin, die Gesellschaft als Organisation zu bestimmen. Dabei wird in der Tradition von Saint-Simon und Weber die Gesellschaft wie eine Organisation behandelt. Dem modernen Staat, häufig als Synonym für Gesellschaft genutzt, werden dabei ähnliche Hierarchien, Zwecksetzungen und Mitgliedschaftsregelungen unterstellt wie Organisationen. Es mag für diese Analyse sowohl bei den bis in die neunziger Jahre existierenden sozialistischen Staaten als auch bei faschistischen Staaten historische Evidenzen geben, für demokratische, auf Gewaltenteilung basierende und über ein autonomes Wirtschafts- und Wissenschaftssystem verfügende Staaten greift dieses Analyseschema aber zu kurz.

Der zweite theoretische Kurzschluss besteht darin, mit dem Postulat einer Organisationsgesellschaft implizit zu unterstellen, dass die Gesellschaft aus Organisationen und nur aus Organisationen besteht (vgl. z. B. die Argumentation bei Perrow 1996). Wissenschaft wird über Universitäten erklärt, und die weitgehend jenseits von Organisationen angesiedelte Verteilung von Reputation durch Publikationen wird ausgeblendet. Der Zugang zur Analyse der Wirtschaft wird über die Funktionsweise von Unternehmen gesucht, Marktprozesse werden dabei aber negiert oder lediglich als Kontextbedingung von Unternehmen aufgefasst. Politik wird über die Funktionsweise politischer Parteien begriffen, und Phäno-

2 Schimank (2001:290) führt als weitere Beispiele einer solchen organisationszentrischen Sicht Merton (1968) und Presthus (1966) an. Ein Revival erlebt die Webersche Tradition sicherlich in der Idee von Ritzer (1997) von einer McDonaldisierung der Gesellschaft (vg). auch Brüsemeister 2000) 
mene wie die Wahl werden lediglich als Wettbewerb zwischen diesen politischen Organisationen konzipiert.

Die Testfrage, die an Theorien angelegt werden muss, ist, wie sie mit dem Verhältnis von Gesellschaft und Organisation umgehen und welche Rolle sie dem Eigenleben von Organisationen, die „,in der Gesellschaft sind“, zugestehen.

\section{Die Gefahr der Auflösung der Organisation in einer allgemeinen Sozialtheorie}

Eine besonders in den sechziger und siebziger Jahren zu beobachtende Reaktion auf die Selbstüberschätzung der Organisationssoziologie war, Gesellschaft nur noch als Kontextbedingung von Organisationen einzuführen. In Kontrast zu Arbeiten in der Tradition von Saint-Simon und Weber wurde ein Teil der Organisationssoziologie, für den am prominentesten der Kontingenzansatz und die Populationsökologie stehen, zu einer ,bescheidenen Disziplin“, die Gesellschaft in einer statischen Form nur noch als Umwelt von Organisationen betrachtete. In Deutschland wurde erst in den neunziger Jahren verstärkt über die Rückkehr der Gesellschaft in die Organisationstheorie diskutiert, und aus verschiedenen Theorieperspektiven wurden Angebote vorgelegt. Die Aussagekraft und die Grenzen dreier prominenter Theorierichtungen seien im Folgenden kurz diskutiert.

Theorie der Strukturierung: Die auf Anthony Giddens zurückgehende Theorie der Strukturierung tritt mit dem nicht gerade bescheidenen Anspruch an, die Alternative zwischen Handlung und Struktur zu überwinden und in einer eigenen Sożialtheorie die wechselseitige Konstitution von Handlung und Struktur zu erklären. Die Dualitât und Rekursivität von Strukturen und Handlungen wird dabei so erklärt, dass das Handeln im Medium von Strukturen erfolgt, also durch Strukturen ermöglicht und beschränkt wird (Strukturiertheit), und eben jene Strukturen zum Resultat hat (Strukturbildung). Dabei werden die von Giddens entwickelten vier Strukturdimensionen Signifikanz, Legitimation, autoritative Ressourcen und allokative Ressourcen übernommen und als Analyseschema für Akteure, Organisationen und Gesellschaft operationalisiert. Mit dieser Konzeptualisierung wird der Anspruch erhoben, in der Organisationsanalyse einerseits einen übertriebenen Voluntarismus und andererseits einen überzogenen Determinismus zu vermeiden. In dieser Konzeptualisierung werden zwischen Akteuren und Organisation sowie Organisationen und Gesellschaft rekursive Schleifen konstruiert, und somit wird ein Zusammenhang zwischen Organisationssoziologie und Gesellschaftstheorie behauptet (vgl. Ortmann 1995: 55; Sydow et al. 1995: 22). Die Herausforderung für die Theorie der Strukturierung besteht darin, nicht nur einen soziologischen Allgemeinplatz vom Zusammenhang von Handlung und Struktur (siehe z.B. für eine identische Argumentation im subjektorientierten Ansatz Bolte 1988) zu einer eigenen Sozialtheorie aufzubauen, sondern eine präzise Bestimmung der Spezifik von Gesellschaft und Organisation vorzunehmen.
Neo-Institutionalismus: Die Stärke des Neo-Institutionalismus ist eine starke Anbindung der Organisation an die Gesamtgesellschaft. Schon in der Arbeit von John Meyer und Brian Rowan (1977) ist darauf aufmerksam gemacht worden, dass Organisationen als soziale Systeme darauf angewiesen sind, über Rationalitätsnachweise oder gar Rationalitätsfiktionen ihre Legitimität in der Gesellschaft herzustellen. Weil das Überleben von Organisationen nicht nur von der Befriedigung technischer Effizienzanforderungen, sondern auch von der Zufriedenstellung institutioneller Erwartungen abhängt, werden Organisationen in der Theorie des Neo-Institutionalismus quasi als Lizenznehmer der Gesellschaft rekonstruiert. Die Schwäche des Neo-Institutionalismus ist, dass ihm über die starke Anbindung der Organisation an gesellschaftliche Erwartungen der Blick für das Eigenleben von Organisationen verloren zu gehen droht. Die Eigenständigkeit der Organisation als soziales System wird im Neo-Institutionalismus über den Entkopplungsbegriff eingeführt. Die Entkopplung dient Organisationen dazu, unterschiedliche Erwartungen aus der Umwelt zu bedienen, ohne an den daraus resultierenden Inkonsistenzen zu zerbrechen. Wie wenig dieser Entkopplungsbegriff aber trägt, wird daran deutlich, dass er in der aktuellen, besonders durch Powell, DiMaggio und Jepperson beeinflussten Diskussion keinen systematischen Platz mehr findet. Die Organisationsperspektive droht dabei dem Neo-Institutionalismus verloren zu gehen.

Institutionenökonomie: Die Institutionenökonomie mit ihren drei Spielarten der Transaktionskostentheorie, der Property-Rights-Theorie und der Principal Agent Theorie geht in einer letztlich Schumpeterschen Tradition von einem methodologischen Individualismus aus. Soziale Systeme wie Unternehmen, Verwaltungen aber auch Staat werden konsequent aus der Perspektive rational handelnder Akteure analysiert. Dem Akteur wird im Rahmen der wahrgenommenen Handlungsrestriktionen und der vor der Entscheidung gebildeten Präferenzen eine individuelle Nutzenmaximierung unterstellt. Institutionen, so die Annahme, entstehen überall dort, wo die Beteiligten durch die Schaffung von Institutionen und die Anpassung ihres Verhaltens an die Institutionen zu einem höheren Nutzenniveau gelangen, als wenn diese Institutionen nicht existieren (vgl. Picot/Reichwald/Wigand 2001: 39; Picot/Dietl/Franck 1999; $39 \mathrm{ff}$.). In der für die Soziologie relevanten Colemanschen Variante werden die Beziehungen zwischen individuellen Akteuren und korporativen Akteuren als asymmetrische Sozialbeziehungen rekonstruiert. Durch die Zunahme des Gewichts von korporativen Akteuren wie Unternehmen und Verwaltungen entstehen neue Risiken für den individuellen Akteur. Als Möglichkeiten zur Resymmetrisierung diskutiert Coleman die paradoxe Eindämmung der korporativen Akteure "Unternehmen" durch die Prozesssteuerung durch den korporativen Akteur "Staat" und den Einbau von Marktelementen (vgl. Coleman 1986: 106 ff.). Die Anfrage an diese Theorierichtung ist, inwiefern es ihr trotz der Fokussierung auf den rational handelnden Akteur gelingt, präzise Konzepte von Institutionen wie Organisation und Gesellschaft zu entwickeln und der Tendenz zu widerstehen, in eine 
Whytesche oder Burnhamsche Konzeptualisierung des Verhältnisses von Akteur und Organisation zu verfallen (vgl. auch Schimank 2000: $241 \mathrm{ff}$.).

$\mathrm{Zu}$ Recht macht Veronika Tacke (2001) auf einen Trend aufmerksam, dass die theoretische Diskussion über Organisation zwar zu elaborierten Sozialtheorien führt, die jeweils den Anspruch haben, die konkurrierenden Sozialtheorien $\mathrm{zu}$ integrieren, die Anlehnung an eine elaborierte Gesellschaftstheorie aber missachtet wird. Schärfer ausgedrückt: Die Gefahr der Theorie der Strukturie rung, des Neo-Institutionalismus und der Institutionenökonomie ist, dass sie sich zwar als Sozialtheorie etablieren können, dass aber sowohl der Begriff der Organisation als auch der der Gesellschaft unterbestimmt bleiben.

\section{Zum Verhältnis von Organisation und Gesellschaft}

Der Zugang, der im Folgenden gesucht wird, nutzt die systematische Bestimmung der Differenz zwischen Gesellschaft und Organisation, um in einem ersten Schritt eine genauere Vorstellung von Merkmalen der Organisationen zu entwickeln (vgl. ausführlich Kühl 2002). Besonders Niklas Luhmann hat herausgearbeitet, dass in der Strukturierung demokratischer, funktional differenzierter Gesellschaften die Bedeutung erstens von Zweeken, zweitens von Mitgliedschaftsinklusionen und - exklusionen und drittens von Hierarchien abnimmt, diese aber als Strukturierungsmerkmal von Organisationen einen zunehmend prominenten Platz erhalten (im Anschluss an Luhmann vgl. auch Kieserling 1994).

Zu Zwecken: Moderne Gesellschaften halten sich im Gegensatz zu den Gesellschiften des Altertums oder des Mittelalters zurück, sich übergeordneten Zwecken wie religiöser Verwirklichung der Staatsbürger oder der Verwirklichung des sozialistischen Menschheitsideals zu verschreiben. Ganz anders Organisationen: Egal ob es sich um eine staatliche Verwaltung, ein Unternehmen oder eine Gewerkschaft handelt - konkrete Zwecke spielen eine zentrale Rolle in der Ausrichtung. Eine Organisation, die völlig auf die Formulierung von Zwecken verzichtet, würde sowohl bei den eigenen Mitgliedern als auch bei der externen Umwelt ein Höchstmaß an Irritation hervorrufen (vgl. Luhmann 1973 : $87 \mathrm{ff}$; 1997: $826 \mathrm{ff}$.).

$\mathrm{Zu}$ Mitgliedschaft als Inklusions- und Exklusionsmechanismus: Auch das Management des Eintritts und Austritts - die Bestimmung von Mitgliedschafter - handhaben Organisationen anders als moderne Gesellschaften. Der Ausschluss von Mitgliedern aus Geselischaften durch Todesstrafe, Verbannung oder Ausbürgerung stellt die Ausnahme dar. Das Management des Einschlusses und Ausschlusses von Mitgliedern ist dagegen ein zentrales Instrument in Organisationen. Über die Bestimmung der Mitgliedschaft kann das Management festlegen, wer zu einem Unternehmen, einer Verwaltung oder einem Verband gehört. Dadurch werden Grenzen geschaffen, in denen sich die Mitglieder (und eben nur die Mitglieder) den Regeln der Organisation zu unterwerfen haben (Luhmann 1995: 16)
Zu Hierarchien: Auch die Hierarchien verlieren in der Gesellschaft an Bedeutung, während sie für die Strukturierung von Organisationen zentral bleiben. Es gibt in den modemen Gesellschaften keinen Herrscher mehr, der über Befehls- und Anweisungsketten in die verschiedenen Lebensbereiche der Bevölkerung hineinregieren kann. Wie das Beispiel Irak in der Hussein-Ära oder Afghanistan zur Zeit der Taliban zeigt, wird eine solche Gesellschaft als rückständig betrachtet. In der modernen Gesellschaft akzeptiert niemand den Kanzler als obersten Vorgesetzten eíner Befehlshierarchie - außer den Mitgliedern des Kanzleramts. Im Gegensatz zu den modemen Gesellschaften sind Organisationen zentral über Hierarchien strukturiert. Erst die Hierarchie stellt sicher, dass die Anweisungen und Zusagen der Spitze auch umgesetzt werden und Organisationen als kollektive Akteure auftreten können (vgl. Luhmann 1997: 834).

Mit diesen markierten Unterschieden steht der Soziologie ein Kategoriengerüst zur Verfügung, das sie für eine präzise Bestimmung der Funktionsweise von Organisationen weiterentwickeln kann. Dabei besteht aus meiner Sicht der Reiz einer Organisationssoziologie, die die Differenz von Organisation und Gesellschaft zum Ausgangspunkt nimmt, in drei Punkten (vgl. auch Strodtholz) Kühl 2002): Erstens erhält die Zweckrationalität, die in der frühen Organisationstheorie eine wichtige Rolle gespielt hat, einen systematisch entwickelten Ort zugewiesen. Sie wird nicht mehr als Ausgangspunkt genommen, sondem als ein Strukturmerkmal von Organisationen rekonstruiert. Zweitens ermöglicht sie eine historische Einordnung des Phänomens der Organisation. Drittens hat man einen präzisen Ausgangspunkt, von dem aus die Kopplungen zwischen den Funktionssystemen der Gesellschaft bestimmt werden können - eine Bestimmung, die die Engführung in ein Konzept von Organisationsgeseilschaft vermeiden kann.

\section{Literatur}

Adorno, Th. W. (1954): Individuum und Organisation. In F. Neumark (Hg.): Individuum und Organisation. Darmstädter Gespräche. Dortmund, S. 21-35.

Altmann, N. et al. (1982): Grenzen neuer Arbeitsformen. Frankfurt a. M./New York.

Altmann, N / Bechtle, G. (1971): Betriebliche Herrschaftsstruktur und industrielle Gesellschaft. München.

Bahrdı, H. P (1982): Die Industriesoziologie - eine „spezielle Soziologie"? In: H.-J. Braczyk et al (Hg): Materialien zur Industriesoziologie. Sonderheft 24 der Köiner Zeitschrift für Soziologie und Sozialpsychologie. Opladen, S 11-15.

Bardmann. Th. M. (1994): Wenn aus Arbeit Abfall wird. Aufbau und Abbau organisatorischer Realitäten. Frankfurt a. M

3 Uberspitzt könnte man argumentieren, dass erst durch das soziale Phänomen der Hierarchie die korporativen Akteure entstehen, die die Rational Choice-Theorie in der Tradition Colemans $(1986 ; 1990)$ braucht, um eine Asymmetrie zwischen Einzelakteuren und korporativen Akteuren zu konzeptualisieren. Siehe besonders Coleman 1993: 9 ff. 
Beck, U. (1982): Folgeprobleme der Modernisierung und die Stellung der Soziologie in der Praxis. In: U. Beck (Hg.): Soziologie und Praxis. Erfahrungen, Konflikte, Perspektiven Göttingen, S. 1-26.

Beckenbach, N (1991); Industriesoziologie: Berlin; New York.

Bolte, K. M. (1988) (Hg.): Mensch, Arbeit und Betrieb. Weinheim.

Böhle, F. (1994) : Negation und Nutzung subjektivierenden Arbeitshandelns bei neuen Formen qualifizierter Produktionsarbeit. In: N. Beckenbach; W van Treeck (Hg.): Umbriche gesellschaftlicher Arbeit. In: Soziale Welt, Sonderband 9, S. 183-206.

Brandt, G. (1990): Arbeit, Technik und gesellschafiliche Entwicklung. Transformationsprozesse des modernen Kapitalismus. Frankfurt a. M.

Brüsemeister, Th. (2000): Die Gesellschaft als organisierte Erwartungs-Enttäuschungs-Spirale - George Ritzers These der McDonaldisierung. In: U. Schimank u. U. Volkmann (Hg.) Soziologische Gegenwartsdiagnosen I. Eine Bestandsaufnahme. Opladen, S. 275-290.

Burnham, J. C. (1948): The Managerial Revolution. What is Happening in the World. Westport CT.

Coleman, J. S. (1986): Die asymmetrische Gesellschaft. Weinheim.

Coleman, J. S. (1990): Foundations of Social Theory. Cambridge.

Coleman, J. S. (1993): The Rational Reconstruction of Society. In: American Sociological Review 58, S. $1-15$.

Daheim, H, / Schönbauer, G. (1993): Soziologie der Arbeitsgesellschaft. Weinheim/München. Eisenstadt, S. N.(1963): The Political Systems of Empires. Glencoe III.

Kieserlìng, A. (1994): Organisationssoziologie und Unternehmensberatung. 6 Lehrvorträge. Bielefeld: unveröff. Ms.

Kieserling, A. (2000): Die Soziologie der Selbstbeschreibung. In: H de Berg u. J. Schmidt (Hg.): Rezeption und Reflexion. Frankfurt a. M., S. 38-92.

Kühl, S. (2002): Sisyphos im Management. Die vergebliche Suche nach der perfekten Organisationsstruktur. Weinheim et al: Wiley.

Kreckel, R. (1993): Theorien sozialer Ungleichheiten im Ubergang. In: R. Kreckel (Hg.): Soziale Ungleichheiten. Soziale Welt, Sonderband 2, S. 3-14.

Latour, B. (1995): Wir sind nie modem gewesen. Versuch einer symmetrischen Anthropologie. Berlin.

Littek, W. / Rammert, W. / Wachtler, G. (1982): Einführung in die Arbeits- und Industriesoziologie. Frankfurt a. M. New York.

Luhmann, N. (1973): Zweckbegriff und Systemrationalität, Über die Funktion von Zwecken in sozialen Systemen. Frankfurt a.M

Luhmann, N. (1995): Funktionen und Folgen formaler Organisation, 4. Aufl., Berlin.

Luhmann, N. (1997): Die Gesellschaft der Gesellschaft, Frankfurt a.M.

Merton, R. K. (1968): Bürokratische Struktur und Persönlichkeit. In: R. Mayntz (Hg.): Bürokratische Organisation. Köln/Berlin, S. 265-276.

Meyer. J. W. / Rowan. B. (1977): Institutionalized Organizations. Formal Structure as Myth and Ceremony. In: American Journal of Sociology 83, S. 340-363.
Offe, C. (1984): Arbeit als soziologische Schlüsselkategorie? In: C. Offe (Hg.): „Arbeitsgesellschaft". Strukturprobleme und Zukunfisperspektiven. Frankfurt a.M./New York, S. 13-43.

Ortmann, G. (1995): Die Form der Produktion. Organisation und Rekursivităt. Opladen.

Ostner, I (2001): Arbeits- und Industriegeselischaft In: G. Kneer, A. Nassehi u. M. Schroer $(\mathrm{Hg}$.): Klassische Gesellschaftsbegriffe der Soziologie. München, S. 55-72.

Perrow, C. (1996): Eine Gesellschaft von Organisationen. In: P. Kenis u. V. Schneider (Hg.): Organisation und Netzwerk. Institutionelle Steuerung in Wirtschaft und Politik. Frankfurt a. M. New York, S. 75-121.

Picot, A. / Reichwald, R. / Wigand, R. T. (2001): Die grenzenlose Unternehmung. Information, Organisation und Management. 4. Aufl. Wiesbaden.

Picot, A. / Dietl, H. / Franck, E. (1999): Organisation. Eine ökonomische Perspektive. 2. Aufl. Stuttgart.

Presthus, R. (1966): Individuum und Organisation. Typologie der Anpassung. Hamburg.

Ritzer, G. (1997): Die McDonaldisierung der Gesellschaft. Frankfurt a. M.

Saint-Simon, C.-H+ (1964): Du Système Industriel. Aalen.

Schimank, U. (2000): Das ,stahlharte Gehäuse der Hörigkeit", revisited - James Colemans "asymmetrische Gesellschaft". In: U. Schimank u, U. Volkmann (Hg.): Soziologische Gegenwartsdiagnosen I: Eine Bestandsaufnahme. Opladen, S. 239-254.

Schimank, U, (2001): Organisationsgesellschaft In: G. Kneer, A Nassehi u. M. Schroer (Hg.): Klassische Gesellschaftsbegriffe der Soziologie. München, S. 278-307.

Schluchter, W. (1985): Aspekte bürokratischer Herrschaft. Studien zur Interpretation der fortschreitenden Industriegesellschaft. Frankfurt a. M

Springer, R. (1997): Krise der Arbeitswissenschaft? Stuttgart: unveroff. Ms.

Strodtholz, P. / Kühl, S. (2002): Qualitative Methoden der Organisationsforschung - ein U. berblick. In: S. Kühl u. P. Strodtholz ( $\mathrm{Hg}$.): Methoden der Organisationsforschung, Ein Handbuch. Reinbek, S. 11-32.

Sydow, J. et al. (1995): Organisation von Netzwerken. Strukturationstheoretische Analysen der Vermittlungspraxis in Versicherungsnetzwerken. Opladen.

Tacke, V. (2001): Einleitung. In: ders. (Hg.): Organisation und gesellschaftliche Differenzierung. Opladen, S. 7-18.

Tacke, V. / Wehrsig, Ch. (1992): Funktionen und Folgen informatisierter Organisationen. In: Th. Malsch a. U. Mill (Hg): ArBYTE. Modernisierung der Industriesoziologie? Berlin, S. $219-239$.

VoB, G. G. / Pongratz. H. J. (1998): Det Arbeitskraftunternehmer. Eine neue Grundform der Ware Arbeitskraft? In: Kölner Zeitschrift für Soziologie und Sozialpsychologie 50 , S. 131-158.

Weber, M. (1972): Wirtschaft und Gesellschaft. 4. Auft. Tübingen.

Whyte, W. H. (1958): Lohn und Leistung. Köln.

Wolf, H. (1999): Arbeit und Autonomie. Ein Versuch über Widersprúche und Metamorphosen kapitalistischer Produktion. Münster. 